\title{
Efectos de la especie invasora Poecilia gillii (Cyprinodontiformes: Poeciliidae) sobre Pseudopoecilia fria en ríos costeros de la región del Chocó, Ecuador
}

\author{
Pedro Jiménez-Prado ${ }^{1,2^{*}}$, Fernando Vásquez ${ }^{1}$, Douglas Rodríguez-Olarte ${ }^{3}$ \& Donald Taphorn ${ }^{4}$ \\ 1. Pontificia Universidad Católica del Ecuador Sede Esmeraldas, Espejo y subida a Santa Cruz, 08010065. Esmeraldas, \\ Ecuador; pedro.jimenez@pucese.edu.ec,.wfvg94@gmail.com \\ 2. Area de Ecología, Departamento de Ciencias Agrarias y del Medio Natural, Escuela Politécnica Superior de Huesca, \\ Universidad de Zaragoza, Carretera de Cuarte s/n, 22071 Huesca, España. \\ 3. Colección Regional de Peces. Museo de Ciencias Naturales. Decanato de Agronomía. Universidad Centroccidental \\ Lisandro Alvarado, UCLA. Barquisimeto, estado Lara, Venezuela; douglasrodriguez@ucla.edu.ve \\ 4.1822 North Charles Street, Belleville, Illinois, 62221, USA; taphorn@gmail.com \\ * Correspondencia
}

Recibido 24-I-2019. Corregido 02-X-2019. Aceptado 15-I-2020.

\begin{abstract}
Effects of the invasive species Poecilia gillii (Cyprinodontiformes: Poeciliidae) on Pseudopoecilia fria in coastal rivers of the Choco region, Ecuador. One of the causes contributing to the decrease of biodiversity is the introduction of exotic species that often end up being invasive, and end up competing with local species. This competition is not always "fair" since invaders commonly have adaptive advantages that give them an advantage over local species. This study was carried out in small watersheds in northwestern Ecuador and identifies the effects of the recent introduction of $P$. gillii on the native species $P$. fria; using population analysis (longitudinal distribution, growth parameters and morphometry). The results show a displacement of $P$. fria towards the upper part of the river, acceleration in its growth and therefore an earlier sexual maturity with a consequent decrease in body size and finally a change in body depth with posterior displacement of the pectoral fin.
\end{abstract}

Key words: invasive species, Pacific, impact.

Jiménez-Prado, P., Vásquez, F., Rodríguez-Olarte, D., \& Taphorn, D. (2020). Efectos de la especie invasora Poecilia gillii (Cyprinodontiformes: Poeciliidae) sobre Pseudopoecilia fria en ríos costeros de la región del Chocó, Ecuador. Revista de Biología Tropical, 68(1), $122-138$

La introducción de especies invasoras puede producir efectos negativos que pueden ir desde la reducción de la diversidad de especies nativas, la alteración del hábitat, la hibridación, la competencia, la depredación y el parasitismo, la aparición de cambios en la estructura de las redes alimenticias comunitarias, el ciclo de nutrientes $\mathrm{y}$, en consecuencia, cambios en el funcionamiento del ecosistema invadido (Gubiani et al., 2018). Sin embargo, la mayoría de las especies introducidas en un nuevo hábitat no logran establecer una población estable (Williamson \& Fitter, 1996), pues su éxito como invasoras depende de superar al menos tres etapas (Deacon \& Magurran, 2016): transporte (posibilidad de llegar a un lugar nuevo), establecimiento (capacidad para adaptarse al nuevo lugar, incluyendo la capacidad reproductiva), y dispersión (capacidad de dispersarse a lo largo de nuevas áreas).

Las características biológicas que facilitan la colonización de nuevos hábitats, como en el 
caso de ciertas especies del género Poecilia, son la ovovivíparidad (Courtenay \& Meffe, 1989), el cuidado parental (Magurran, Seghers, Carvalho, \& Shaw, 1992) y la formación de cardúmenes (Camacho-Cervantes, García, Ojanguren, \& Magurran, 2014a; CamachoCervantes, Ojanguren, Deacon, Ramnarine, \& Magurran, 2014b); además, por ejemplo, las hembras de $P$. reticulata tiene la capacidad de almacenar esperma hasta por 10 meses (LópezSepulcre, Gordon, Paterson, Bentzen, \& Reznick, 2013), lo que le permite a una hembra, por si misma, colonizar una nueva localidad, y fundar una población estable (Deacon, Ramnarine, \& Magurran, 2011; Deacon \& Magurran, 2016). Algunos autores (Meyer, Salzburger, \& Schartl, 2006; Lampert \& Schartl, 2008) reportan la hibridización entre especies emparentadas de Poecilia, lo que a su vez podría incrementar el riesgo de extinción para las especies locales (Magalhães \& Jacobi, 2017). Se ha demostrado que los poeciliidos han sido la causa de la disminución en las poblaciones de algunos cyprínidos endémicos de Norteamérica (Magurran, 2009). Los efectos negativos de algunas especies de Poecilia como organismo invasor, van desde la competencia por los alimentos hasta el acoso sexual a las hembras de otras especies por parte de los machos (Valero, Macías-García, \& Magurran, 2008). Aunque, específicamente sobre $P$. gillii, se conoce poco, se sabe que no solo es una de las especies más abundantes en ríos de Centro América (Bussing, 1998; Rodríguez-Pérez \& Contreras, 2012), sino también que se trata de una especie euritrópica de alta tolerancia (Barquero-Gonzáles, Mora-Vargas, ValerioVargas, \& Ulloa-Rojas, 2018), estenofágica, especializada en consumo de algas filamentosa y microalgas, con escaso consumo de componente animal (Morales \& García-Alzate, 2016), incluso con adaptación y poca sensibilidad a condiciones de contaminación por pesticidas (Mena et al., 2014).

La distribución natural de $P$. gillii (Kner 1863) en Centroamérica, en su vertiente atlántica va desde Guatemala hasta Colombia y en su vertiente Pacífica va desde Guatemala hasta
Panamá (Froese \& Pauly, 2018); pero al igual que algunos poecílidos utilizados para el control de plagas y/o para la acuariofilia, se distribuyen también en localidades muy lejanas a su origen, como es el caso incluso de las islas Fiyi o en Oahu en Hawái (registros de University of Michigan Museum of Zoology, 2018).

Un registro de la introducción de ésta especie se ha dado recientemente en el río Atacames, noroccidente de Ecuador, donde hasta el año 2012, solo habitaba P. fria (Jiménez-Prado, 2012). Ante la presencia actual de $P$. gillii en el río Atacames, el presente trabajo analiza sus características poblacionales y morfológicas, cuáles son los efectos que esta especie está generando en la especie nativa $P$. fría y si existe alguna evidencia de hibridación. La combinación de los datos en la relación talla peso, valores de crecimiento-edad y forma del cuerpo aportan con información nueva sobre el efecto que produce la introducción de especies. Se trata de testar la hipótesis de que la competencia entre $P$. gillii y $P$. fria podría ocasionar desplazamiento altitudinal (Cucherousset \& Olden, 2011) y cambios en la forma del cuerpo en la población nativa (Bourke, Magnan, \& Rodriguez, 1999; Latta, Bakelar, Knapp, \& Pfrender, 2007; Fisk, Latta, Knapp, \& Pfrender, 2007).

\section{MATERIALES Y MÉTODOS}

La región noroccidental ecuatoriana presenta características de alta precipitación y montañas bajas que permiten la formación de pequeñas cuencas hidrográficas que mantienen corrientes permanentes durante todo el año y que drenan hacía el océano Pacífico, como son las de los ríos contiguos de Atacames y Súa (Fig. 1). El origen de estos ríos presenta altitudes no mayores a los $200 \mathrm{msnm}$, una extensión del cauce cercana a los $40 \mathrm{~km}$ y un área de drenaje con alrededor de los 300 $\mathrm{km}^{2}$. Estas cuencas son caracterizadas por la presencia de pastos y cultivos en un $79.5 \%$, zona urbana un 3.2 y parches de bosque natural intervenido en un $17.3 \%$. El área de estudio se caracteriza por tener un verano, que va de julio 


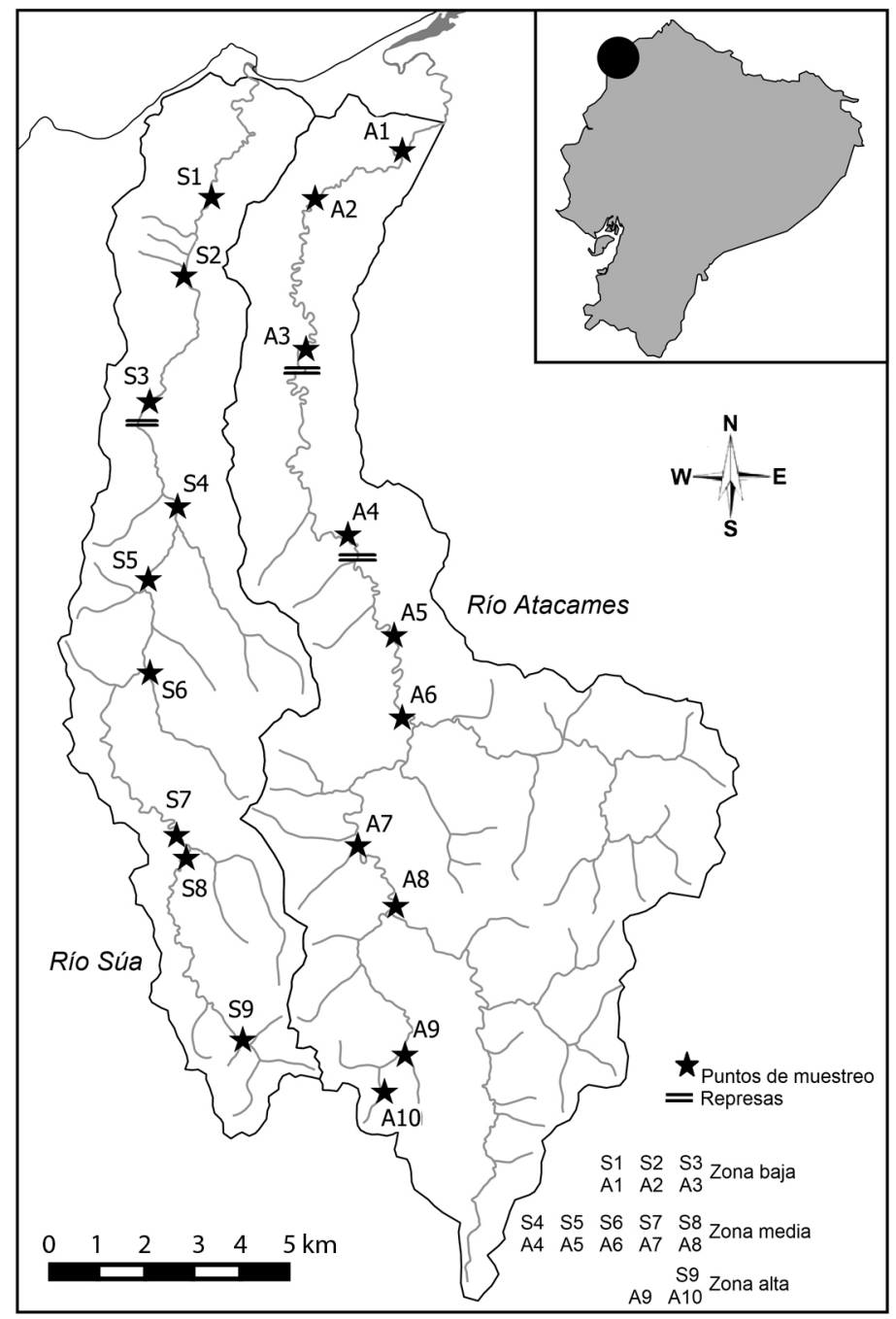

Fig. 1. Área de estudio con localidades de muestreo, separación por zonas de estudio y presencia de represas.

Fig. 1. Study area with sampling locations, separation by study zones and reservoirs.

a diciembre, con precipitaciones esporádicas y escasas $(132 \mathrm{~mm})$ y temperatura ambiental promedio de $25.2{ }^{\circ} \mathrm{C}$ (mínima $=20.0$; máxima $=38.0$ ); mientras que en el invierno las lluvias son esporádicas pero abundantes $(459.6 \mathrm{~mm})$ y la temperatura promedio alcanza $\operatorname{los} 27.1^{\circ} \mathrm{C}$ (mínima $=21.0 ;$ máxima $=37.0)$. En los dos ríos existen presas ubicadas en la parte media del cauce y que constituyen una barrera artificial entre las diferentes zonas de los ríos.

Los ríos Atacames y Súa están sujetos a condiciones ambientales semejantes, por lo que los parámetros poblacionales de $P$. fria del río Súa pueden servir como referencia para identificar los cambios en los parámetros poblacionales de $P$. fria del río Atacames, en presencia de P. gillii. Con base en lo anterior, desde julio 2016 a junio 2017 se realizaron muestreos bimensuales a lo largo de los ríos Atacames, en 10 localidades distribuidas más o menos de forma equitativa a lo largo del cauce principal y Súa nueve localidades distribuidas más o menos de forma equitativa a lo largo del cauce principal (Fig. 1). Para cada 
localidad de muestreo se levantó información físico-química del agua in situ: temperatura, $\mathrm{pH}$, conductividad, oxígeno disuelto, (con sensor multiparamétrico Hanna HI9829), turbidez, sólidos en suspensión dureza, amonio, nitratos y fosfatos (con colorímetro Hach DR900). La recolección de peces se la hizo a pie con una red de arrastre (6 m de largo x $1.20 \mathrm{~m}$ de ancho y $3 \mathrm{~mm}$ de distancia entre nudo), con un esfuerzo de captura acumulado (CPUE) estandarizado a $60 \mathrm{~m}^{2}$ y dos hombres por cada localidad de muestreo. Como el hábitat natural de $P$. gillii y $P$. fria es bentopelágico en vados y sus orillas, el arrastre garantizó la recolecta sino de todo, de la gran mayoría de individuos que habitaban en esa unidad de muestreo.

En el río Atacames $P$. gillii presenta patrones de coloración propios de $P$. fria (Fig. 2), lo cual sugirió inicialmente una posible hibridación, para comprobar o descartar este supuesto se implementó un análisis por morfometría geométrica para identificar y cuantificar la variación en la forma del cuerpo, entre las especies y entre los ríos, a través de representaciones visuales de las diferencias de forma corporales (Toro, Manríquez, \& Sauzo, 2010) y así valorar cuan cercanas o diferentes eran; es decir, si existía o no evidencia de hibridación.

Análisis de los datos: para diferenciar la dinámica ambiental a lo largo del gradiente altitudinal en los dos ríos, las localidades de muestreo fueron agrupadas según sus características geomorfológicas y altura sobre el nivel del mar, lo que permitió dividir los cauces en tres zonas: alta, media y baja (Tabla 1). Se analiza entonces la distribución de las especies, a lo largo de las tres zonas de ambos ríos, para evaluar su abundancia relativa.

Parámetros ambientales: con la finalidad de comparar los parámetros ambientales entre los ríos, luego de comprobar los supuestos de normalidad y homocedasticidad y con un $95 \%$ de confianza, se aplicó inicialmente a una $\mathrm{t}$ de student y luego un ANOVA para las zonas (alta, media y baja) en y entre los ríos. El ordenamiento de los diferentes parámetros en los dos ríos se realizó mediante un análisis de componentes principales (ACP); y finalmente para agruparlos por su similaridad, se realizó un análisis de conglomerados jerárquicos con el método Ward y distancias con correlación de Pearson a partir de medidas de similitud (1-S) y variables estandarizadas (Balzarini et al., 2008).

Para la distribución y los parámetros de crecimiento de los peces: se analiza la distribución espacial y la abundancia relativa de las dos especies a lo largo del gradiente altitudinal (zonas alta, media y baja), en los dos ríos. Por otro lado, con el total de los organismos recolectados en cada río y con la finalidad de obtener valores descriptivos sobre la edad de los peces, se calculó los parámetros de crecimiento (K) y de longitud teórica asintótica $\left(\mathrm{L}_{\infty}\right)$; estos

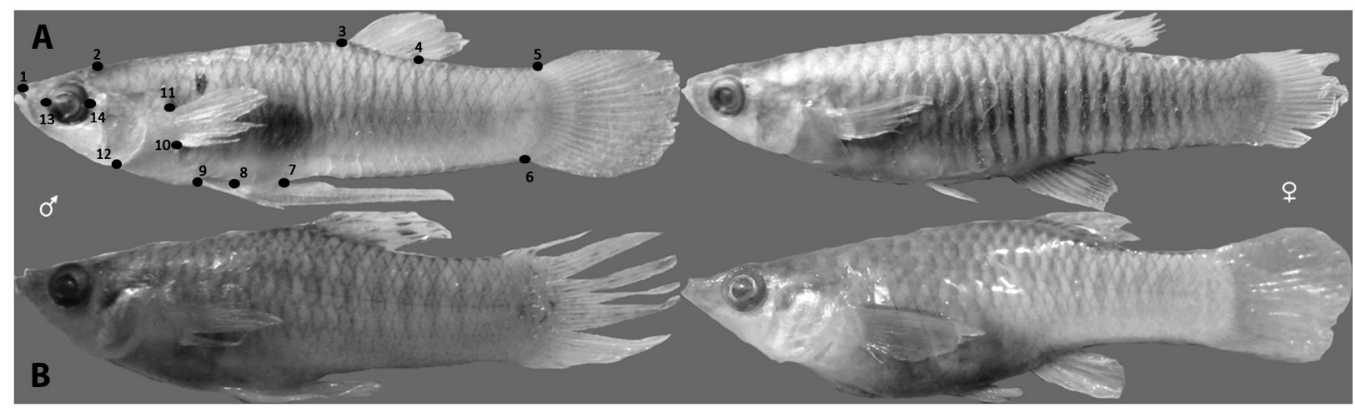

Fig. 2. Pseudopoecilia fría (a) y Poecilia gillii (b). Izquierda: machos, derecha: hembras. El primer ejemplar muestra los hitos empleados en el análisis de morfometría geométrica para todos los ejemplares.

Fig. 2. Pseudopoecilia fria (a) and Poecilia gillii (b). Left: males, right: females. The first specimen shows the milestones used in the geometric morphometric analysis for all the specimens. 
苞

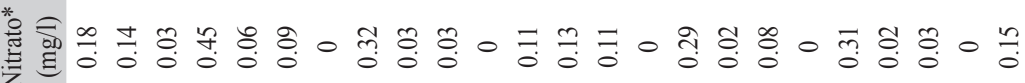

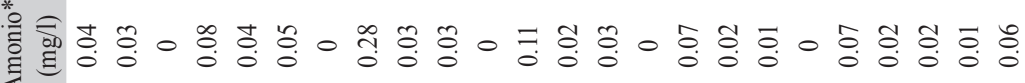
$\stackrel{0}{\circ}$

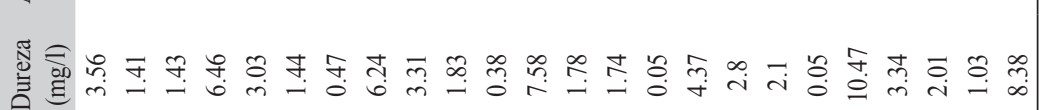
害疍

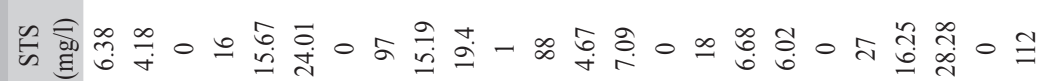
ธิํำ \%

苑 웅 㟒雚 号

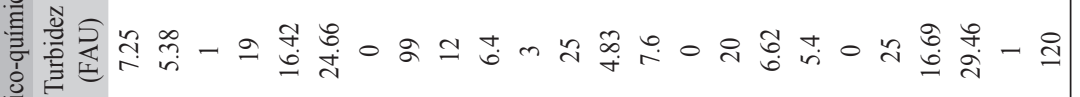
急

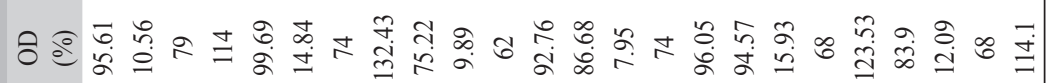

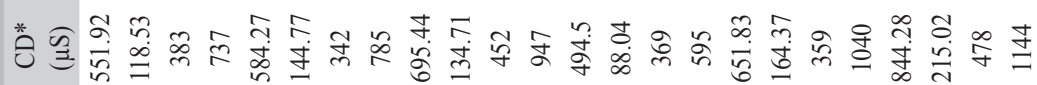

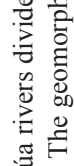
牙泀通 is 1

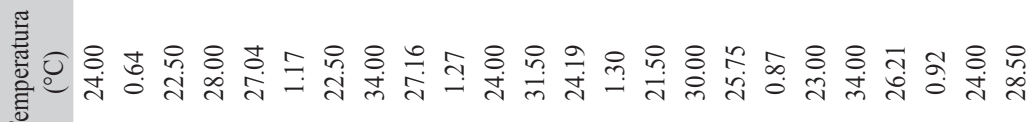

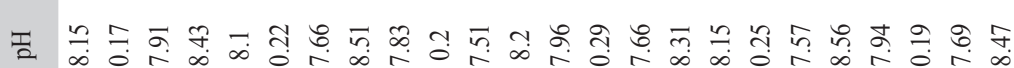

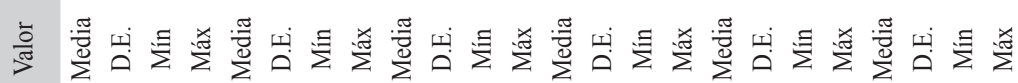

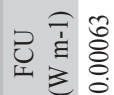

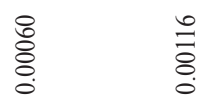

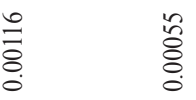
瓷

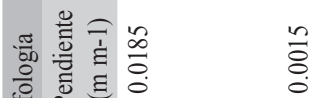

$\frac{2}{8} \quad \frac{8}{0} \quad$

$\stackrel{n}{8}$

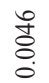

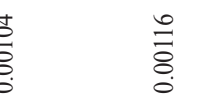

递苛

$$
\underset{q}{\stackrel{q}{g}}
$$

0
0

年

홍.

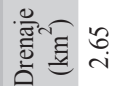

$\stackrel{\circ}{6}$

$\infty$
$\dot{n}$
$\dot{n}$

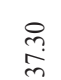

$\infty$
$\stackrel{\sim}{~}$

$\stackrel{\infty}{i}$

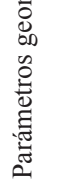

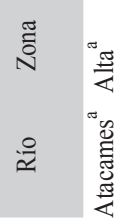

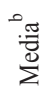

高

nn

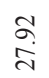

$\underset{n}{n}$

ॠ

氜

莡

姿

0
0
0
0
0
0
0
0
0
0
0
0
0
0
0
0
0
0

\section{î} $e$
$n$
$b$
2 
fueron obtenidos con el método Elefan I (Pauly \& David, 1981), que se basa en el desplazamiento modal de secuencias temporales de datos de longitud y que permiten elaborar la curva de crecimiento de von Bertalanffy (Sparre \& Venema, 1992): $\mathrm{Lt}=\mathrm{L}_{\infty}\left(1-\mathrm{e}^{-\mathrm{K}(\mathrm{t}-\mathrm{to})}\right)$. Según esta expresión los peces crecen en promedio, hacia la longitud asintótica $\left(\mathrm{L}_{\infty}\right)$, a una tasa de crecimiento $(\mathrm{K})$ y en un tiempo $(\mathrm{t})$. La edad teórica al nacimiento $\left(\mathrm{t}_{0}\right)$ fue calculada a través de la fórmula empírica: $\log \left(-\mathrm{t}_{0}\right)=-0.3922-$ $0.2752 \log$ L $\infty-1.038 \log \mathrm{k}$ (Pauly, 1979).

Para calcular el coeficiente de mortalidad natural (M) se utilizó la fórmula empírica: $\operatorname{Ln}(\mathrm{M})=-0.0066-0.279 \log _{10} \mathrm{~L}_{\infty}+$ $0.6543 \log _{10} \mathrm{~K}+0.4634 \log _{10} \mathrm{~T}$ (Pauly, 1983); para lo cual se requiere los parámetros de crecimiento ( $\mathrm{L} \infty \mathrm{y} \mathrm{k})$ y la temperatura media $\left({ }^{\circ} \mathrm{C}\right)$ del ambiente en que las especies viven (para este estudio se empleó el valor de 25.7 ${ }^{\circ} \mathrm{C}$, que es la temperatura anual promedio del agua en estos ríos). Finalmente, el índice de rendimiento del crecimiento ( $\left.\varphi^{\prime}\right)$ que se calculó según la ecuación: $\left(\varphi^{\prime}\right)=\log _{10} k+2 \log _{10} \mathrm{~L} \infty$ (Pauly \& Munro, 1984). Este índice permite entender cuán diferentes son las curvas de crecimiento entre $P$. fria (en los ríos Atacames y Súa) y $P$. gillii.

Para el análisis de la forma de los peces: Con la finalidad de garantizar la mayor variabilidad, se seleccionaron al azar especímenes recolectados a lo largo de todas las zonas y en todos los periodos de muestreo, hasta lograr un n mínimo de 30 individuos; se analizaron finalmente 37 de $P$. gillii, 34 de $P$. fria del río Atacames y 61 de $P$. fria del río Súa. Se tomaron fotografías de cada individuo con una cámara digital (Nikon D5100 24MP), que generaron archivos tipo "TPS" en el programa tpsUtil (Tps_Utility. Rohlf, 2018a), donde se recogieron coordenadas bidimensionales para 14 puntos de referencia (hitos), digitalizados en cada espécimen mediante el programa tpsDig2 (Tps_Digitize. Rohlf, 2018b). Los 14 hitos analizados generaron un total de 24 variables numéricas, correspondientes a los ejes $\mathrm{X}$ y Y de cada hito analizado, menos cuatro que se elimina en el proceso (Fig. 2). Los datos obtenidos se alinearon a través de la superposición de Procrustes a través del programa "TpsRelw32" (Tps_Relative Warps. Rohlf, 2018c), de esta manera se elimina la variación relacionada con la rotación, la traslación y el tamaño. Mediante la técnica de "placa delgada" (Thompson, 1917), se crearon configciones de consenso para cada una de las formas entre especies.

Para entender los principales patrones de divergencia en la forma pura del cuerpo entre muestras se aplicó un análisis de variables canónicas (AVC) mediante el programa MorphoJ 2.0 (Klingenberg, 2011). Este análisis se empleó inicialmente para encontrar las características de forma que mejor distingan a los grupos predefinidos de especímenes ( $P$. gillii$P$. fria del río Atacames y $P$. fria del río Súa). Por la heterogeneidad en el tamaño, entre las muestras, los datos de la forma del cuerpo se corrigieron mediante una regresión en cada grupo, a partir de los datos de la forma del cuerpo, con el $\log _{10}$ del tamaño "centroide" $\left(\mathrm{TC}=\sqrt{ } \Sigma\left(\mathrm{d}^{2}{ }_{1}+\mathrm{d}^{2}{ }_{2} \ldots \mathrm{d}^{2}{ }_{\mathrm{n}}\right)\right.$, pero en este caso los residuos de la regresión se usaron para realizar un nuevo AVC. La configción de hitos que representan la divergencia de la forma del cuerpo a lo largo de la variable canónica 1 (VC1) y la variable canónica 2 (VC2) son mostrados para proporcionar una representación visual de las diferencias en la forma del cuerpo asociadas con la distribución de los puntos en la figura. Para garantizar que las muestras pertenecientes a zonas diferentes del río no fueran excluyentes entre sí, se repitió este procedimiento para cada una de ellas; sin embargo, arrojó patrones similares, por lo que se presenta resultados conjuntos para evitar reiteraciones. Para entender específicamente cual es la diferencia en la forma del cuerpo de $P$. fria del río Atacames y la forma del cuerpo de $P$. fria del río Súa, los datos también fueron sometidos a un análisis discriminante (AD) pareado entre ríos; el factor de escala para la figura resultante de la placa delgada se amplió a $2 \mathrm{X}$ con la finalidad de resaltar las diferencias. Todos los valores obtenidos en la morfometría (24 variables) fueron analizados con MANOVA y prueba de 
Hotelling con nivel corregido por Bonferroni para la comparación pareada. Para los análisis estadísticos fue utilizado el programa Infostat (Di Rienzo et al., 2019) y PAST 2.17, mientras que para la obtención de los parámetros de crecimiento se utilizó FISAT II (Gayanilo, Sparre, \& Pauly, 1996).

\section{RESULTADOS}

Para los parámetros ambientales: El análisis de los parámetros ambientales, entre zonas y por ríos, muestra que existen diferencias significativas entre ellas para el río
Atacames (Wilks $=0.04, \mathrm{~F}=20.10: 20(96), \mathrm{P}$ $<0.001$ ) y también para el río Súa (Wilks = $0.14, \mathrm{~F}=6.97: 20(84), \mathrm{P}<0.001)$; y aunque la comparación de cada parámetro ambiental, analizado de forma independiente entre los ríos, mostró diferencias significativas para las variables de conductividad, amonio, nitrato y fósforo (Tabla 1), el análisis de componentes principales mostró una clara relación entre zonas bajas con mayor temperatura, turbidez, dureza, conductividad, sólidos totales y fosfatos, mientras que las zonas medias y altas estaban caracterizadas por presentar mayor $\mathrm{pH}$, oxígeno disuelto y nitratos (Fig. 3A),
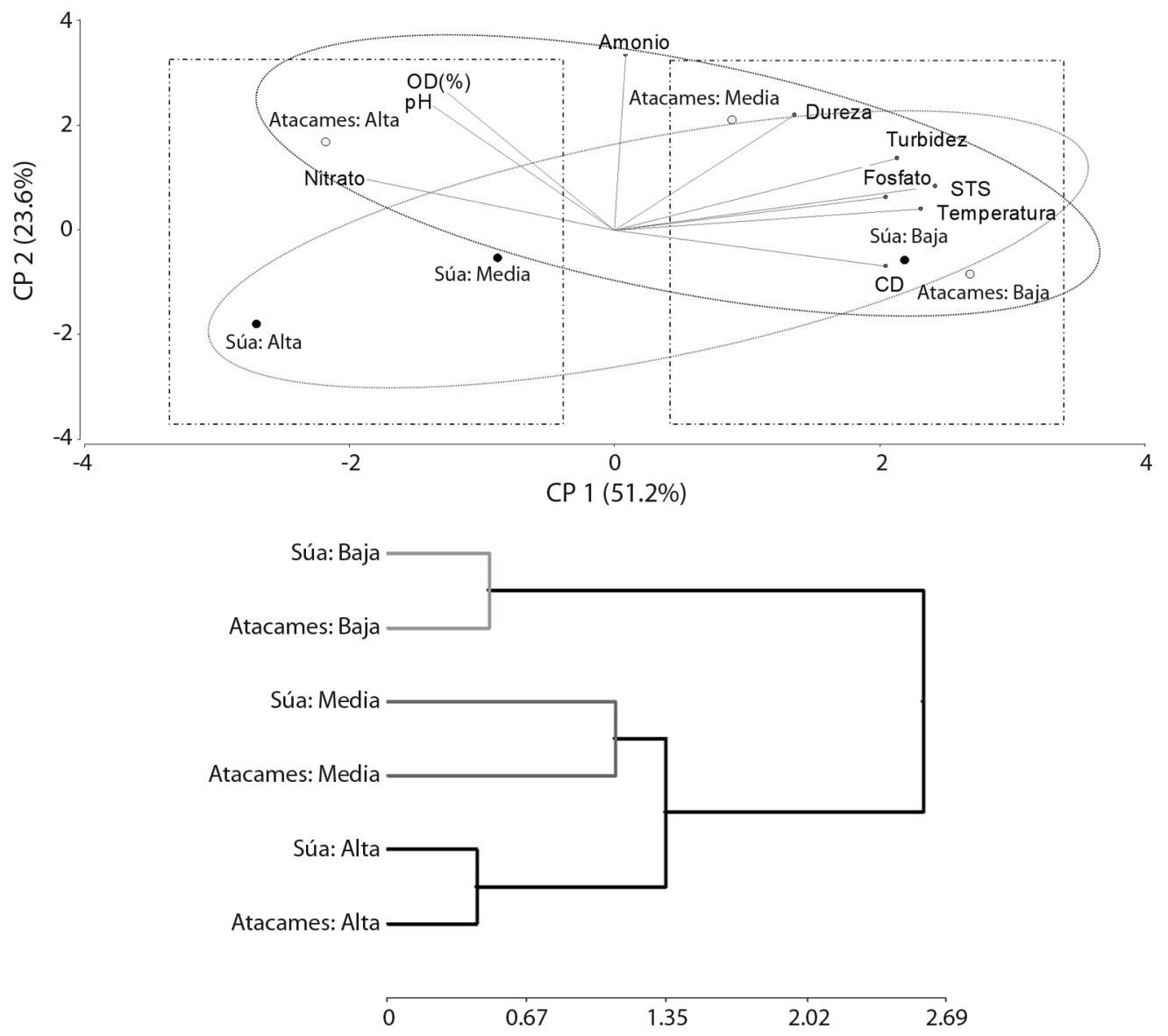

Fig. 3. Análisis de componentes Principales entre los parámetros físico-químicos del agua y las diferentes zonas de los ríos Atacames y Súa (A) y análisis de conglomerados entre las diferentes zonas en cada río (B).

Fig. 3. Principal component analysis between the physical-chemical parameters of water and different zones of Atacames and Súa rivers (A), Conglomerate analysis between the different zones in each river (B). 


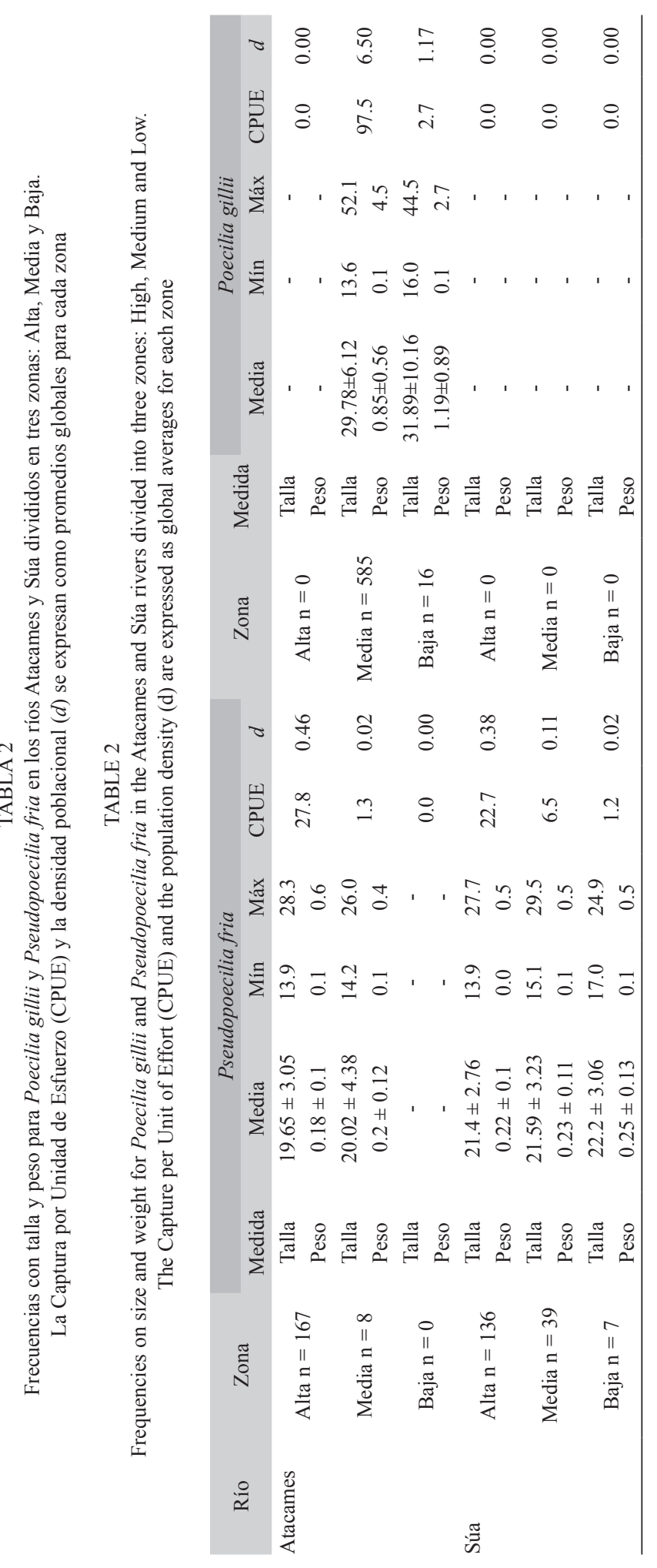


TABLA 3

Parámetros de edad y crecimiento para la población de Pseudopoecilia fria del río Atacames y del río Súa; así como para Poecilia gillii, presente solo en el río Atacames

TABLE 3

Parameters of age and growth for the population of Pseudopoecilia fria and P. gillii from the Atacames and Súa rivers ( $P$. gillii was present only in the Atacames River)

\begin{tabular}{lcccccc} 
& $\mathrm{K}\left(\mathrm{año} \mathrm{o}^{-1}\right)$ & $\mathrm{L}_{\infty}(\mathrm{mm})$ & $\mathrm{t}_{\mathrm{o}}$ & $\phi^{\prime}$ & $\mathrm{M}\left(\mathrm{año} \mathrm{o}^{-1}\right)$ & $\mathrm{Lm} 50(\mathrm{~mm})$ \\
Poecilia gillii & 0.16 & 66.0 & -0.857 & 2.84 & 0.42 & 44.0 \\
Pseudopoecilia fria en Atacames & 0.15 & 31.5 & -1.124 & 2.17 & 0.49 & 21.0 \\
Pseudopoecilia fria en Súa & 0.10 & 37.45 & -1.635 & 2.14 & 0.36 & 24.8 \\
\hline
\end{tabular}

$\mathrm{K}=$ Tasa de crecimiento; $\mathrm{L}_{\infty}=$ Longitud asintótica; $\mathrm{T}_{0}=$ Edad teórica al nacimiento; $\phi$ ' $=$ Rendimiento para índice de crecimiento; $\mathrm{M}$ = mortalidad; Lm50 = Edad calculada de la primera maduración.

$\mathrm{K}=$ Growth rate; $\mathrm{L}_{\infty}=$ Asymptotic length; $\mathrm{T}_{0}=$ Theoretical age at birth; $\phi$ ' = Performance for growth rate; $\mathrm{M}=$ mortality; Lm50 $=$ Calculated age of the first maturation.

el componente 1 reúne el $51.2 \%$ total de la varianza y el componente 2 el $23.6 \%$; por lo que al realizar el análisis de conglomerados, las zonas se muestran clasificadas de forma que sus condiciones muestran similitud, con una correlación de 0.84 , entre ambos ríos (Fig. 3B).

\section{Para la distribución y el crecimiento de} las especies: en el río Atacames, la especie introducida $P$. gillii fue predominante en la zona media, y escasa en la zona baja, mientras que la especie nativa $P$. fria se la registra predominantemente en la zona alta. En el río Súa habita únicamente la especie nativa y con mayor frecuencia en la zona alta (Tabla 2). En todos los casos la especie introducida tuvo mayor talla y peso que la especie nativa. La talla de P. fria mostró diferencias significativas entre los ríos Atacames y Súa ( $\mathrm{F}=348.7$ :2(955), $\mathrm{P}<$ 0.001 ), mayores para el primer río; sin embargo, al comparar sus pesos hubo igualdad ( $\mathrm{F}=$ 1.25:2(955), $\mathrm{P}>0.05)$.

La presencia conjunta de $P$. gillii y $P$. fria se registra exclusivamente en una localidad de la zona media, precisamente aquella que está encerrada entre las dos represas, lo que a su vez podría estar restringiendo su movilidad y permitiendo, tal vez el acceso a la parte inferior del río, pero seguramente impidiendo el acceso a la parte superior. Esto se evidencia porque $P$. fria no está ni en la parte inferior (zona baja) ni en cuatro localidades de muestreo por encima de la represa (también parte de la zona media).

El análisis de edad y crecimiento mostró que $P$. gillii tiene el mayor índice de crecimiento $\left(0.16\right.$ año $\left.^{-1}\right)$, similar a $P$. fria de Atacames $\left(0.15\right.$ año $\left.^{-1}\right)$; sin embargo, ambos son superiores al de $P$. fria del Súa $\left(0.10\right.$ año $\left.{ }^{-1}\right)$; algo equivalente sucede con la mortalidad calculada, donde $P$. gillii y $P$. fria del Atacames tienen un índice similar entre ellos, pero superior al de $P$. fria del Súa $\left(0.42\right.$ año $^{-1}$ y 0.49 año $^{-1}$ Versus 0.36 año $\left.{ }^{-1}\right)$. La longitud asintótica calculada para P. fria del Atacames $\left(\mathrm{L}_{\infty}=31.5 \mathrm{~mm}\right)$ resultó ser inferior al de $P$. fria del Súa $\left(\mathrm{L}_{\infty}=37.45\right.$ $\mathrm{mm}$ ); pero, por otro lado, su madurez calculad se produce antes (a los $21 \mathrm{~mm}$ Vs. $24.8 \mathrm{~mm}$ ). A través de su índice de rendimiento se puede ver que sus curvas de crecimiento son muy similares $\left(\phi^{\prime}=2.17\right.$ Vs. 2.14), (Tabla 3). Hay que resaltar que el cruce entre crecimiento y madurez de $P$. fria, entre la población del río Atacames y la del río Súa, se produce cuando la primera madura antes y por lo tanto detiene su crecimiento mientras que la segunda alcanza su madurez con mayor edad, por lo que logra un crecimiento mayor (Fig. 4).

Para el análisis de la forma de los peces: el análisis de variable canónica demostró que las dos especies de poecílidos están morfológicamente aislados, por lo que es poco probable 


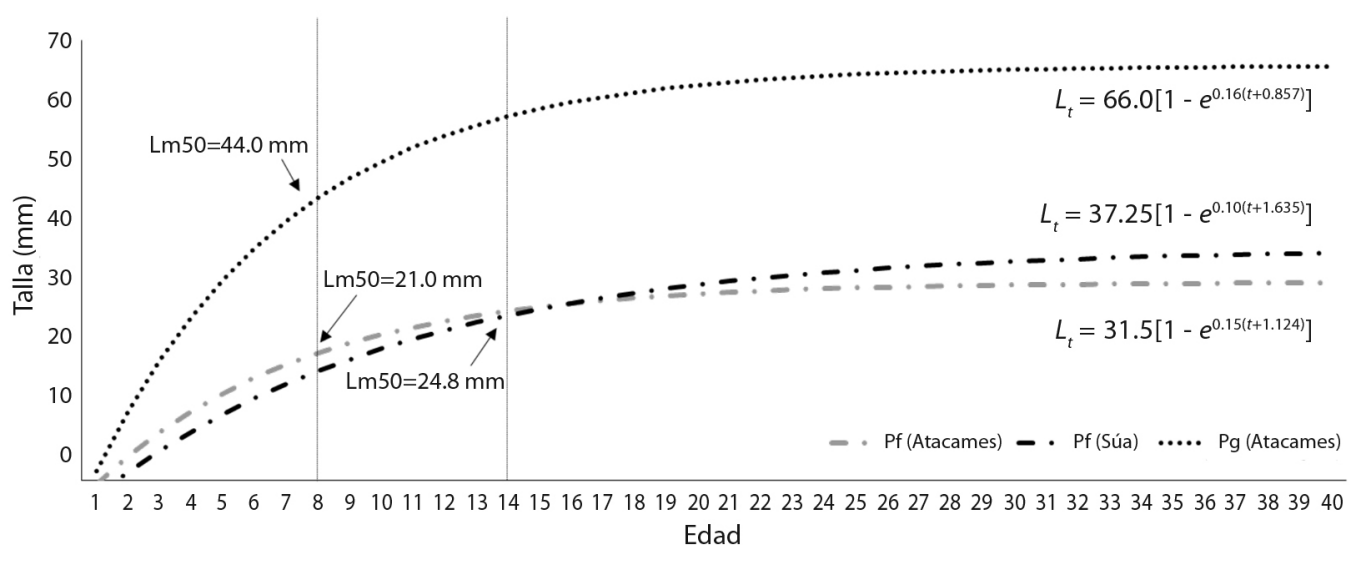

Fig. 4. Curvas de crecimiento de Pseudopoecilia fria (Pf) y Poecilia gillii (Pg) en los ríos Atacames y Súa.

Fig. 4. Growth curves of Pseudopoecilia fria $(\mathrm{Pf})$ and $P$. gillii $(\mathrm{Pg})$ from the Atacames and Súa rivers.

que hayan formado híbridos, esto se manifiesta en el VC1 y por el MANOVA generado por las variables de cada hito (Wilks $=0.10, \mathrm{~F}=$ 20.96, $\mathrm{P}<0.001$ ); pero además, entre $P$. fria del Atacames y $P$. fria del Súa, también se puede ver que existen diferencias a través del VC2 y por la prueba de comparación pareada (Wilks $=0.0085, \mathrm{~F}=35.83, \mathrm{P}<0.001$ ) (Fig. 5), donde el componente uno reúne el $65.0 \%$ de la varianza total y el componente 2 el $23.7 \%$. El análisis discriminante entre $P$. fria del Atacames y P. fria del Súa mostró como significativa la diferencia entre sus formas $(\mathrm{T}=219.44$, $\mathrm{P}$ $<0.001)$. El cuerpo de P. fria del Atacames es ligeramente más alto, pero no más largo que $P$. fria del Súa; sin embargo, la posición de las diferentes estructuras, señalados por los diferentes hitos, se mantienen al mismo nivel, es decir sin desplazamiento de tipo anterior ni posterior. Pero, la característica disímil más clara es la inserción de la aleta pectoral, de $P$. fria del Atacames, que se encontró por detrás de la inserción de la aleta pectoral en P. fria del Súa (Fig. 6).

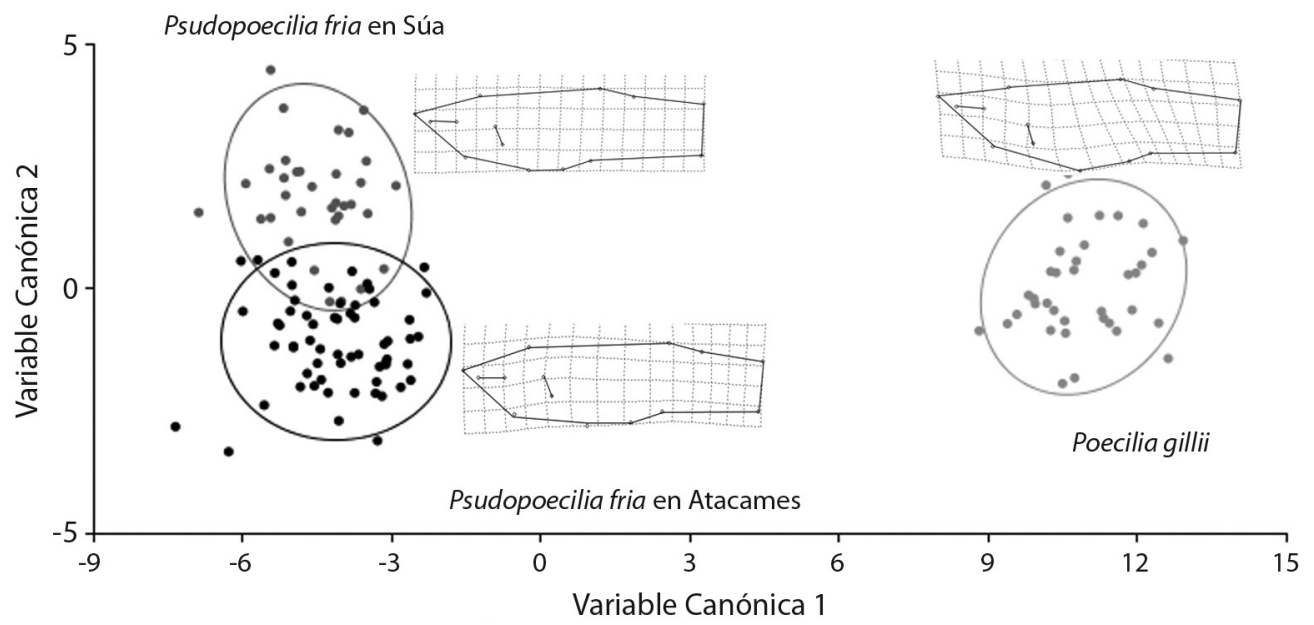

Fig. 5. Análisis de Variables Canónicas de la forma del cuerpo de Pseudopoecilis fria y P. gillii en los ríos Atacames y Súa. Fig. 5. Canonic variable analysis of the body form of Pseudopoecilis fria and P. gillii from the Atacames and Súa rivers. 


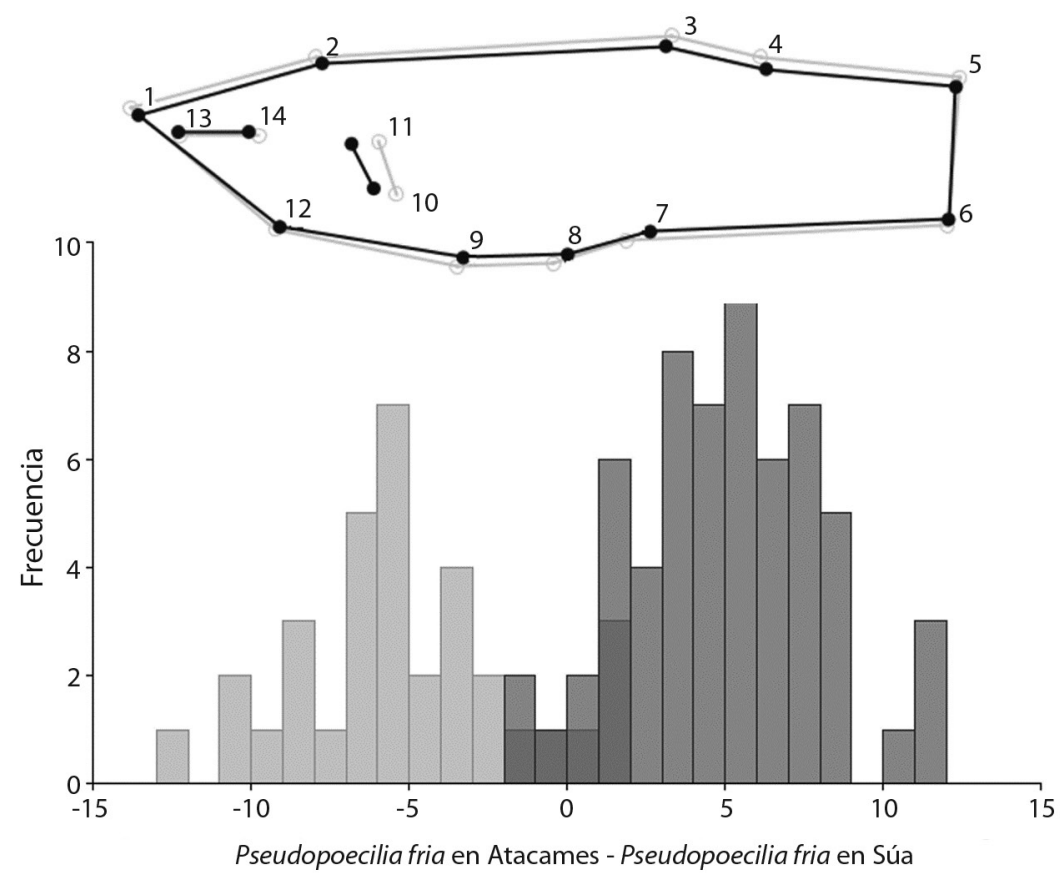

Fig. 6. Análisis discriminante de la forma del cuerpo de Pseudopoecilia fria entre los ríos Atacames y Súa. Fig. 6. Discriminant analysis of the body shape of Pseudopoecilia fria between Atacames and Súa rivers.

\section{DISCUSIÓN}

Los especímenes recolectados entre las represas, fueron pescados en el mismo esfuerzo de captura, lo que evidencia que si pueden forman grupos heteroespecíficos, al menos en esa área. Se sabe que los animales se asocian cuando el "proyecto conjunto" (por ejemplo, buscar comida, explorar el entorno o evitar depredadores) brinda beneficios a todos los organismos involucrados (Leimar \& Hammerstein, 2010); por ejemplo, cuando las decisiones de asociarse en grupo están influenciadas por los niveles percibidos de riesgo y/o a causa de la competencia por los recursos (Mathis \& Chivers, 2003).

Además, las especies recién introducidas en un nuevo hábitat se encuentran en bajas densidades, haciéndolos vulnerables debido a las desventajas de formar un grupo pequeño. Por ejemplo, el tomar más tiempo para localizar los alimentos e incluso el pasar menos tiempo alimentándose (Taylor \& Hasting, 2005), por lo que una asociación heteroespecífica podría mejorar las condiciones de supervivencia cuando, como en este caso, resultan en una desventaja ante el nuevo ambiente (Stephen \& Sutherland, 1999). Específicamente, se ha demostrado que $P$. gillii tiende a asociarse con especies nativas, para obtener beneficios, sobre todo en las primeras etapas de una introducción (Camacho-Cervantes et al., 2014a, b; Camacho-Cervantes, Ojangure, \& Magurran, 2015). Se desconoce si los nativos muestran una tendencia mutua a asociarse con esta especie o si las asociaciones heteroespecíficas ocurren debido a la imposición de una especie sobre la otra (Camacho-Cervantes, Ojanguren, Domínguez-Domínguez, \& Magurran, 2018). Pero al mismo tiempo, esa disposición o imposición sobre las especies nativas para interactuar con especies invasoras podría estar asociada con un rasgo ambiental clave que aumenta la vulnerabilidad local a la invasión, 
al ayudar a individuos fundadores a atenuar las desventajas de ser parte de una población pequeña (Camacho-Cervantes et al., 2018). Así, el éxito de la invasión depende de encontrar un momento o lugar donde los invasores puedan establecerse y, en algunos casos, superar a las especies residentes (Shea \& Chesson, 2002), como parece ser que ha sucedido en todo el resto de la zona media del río Atacames, donde el dominio de $P$. gillii es absoluto, desplazando o aislando a $P$. fria hacía la zona alta.

En este estudio, parece ser que luego del beneficio que pudo haberles significado el asociarse cuando llegó la especie introducida, la presión ejercida por $P$. gillii se manifiesta en el aislamiento de $P$. fria hacia la zona alta. Existe evidencia de este tipo de desplazamiento; por ejemplo, en Perú, en toda la cuenca baja del Río Grande, en la región costera de Ica Ortega, Guerra y Ramírez (2007) señalan la presencia exclusiva de Oreochromis niloticus y $P$. reticulata durante los 10 primeros kilómetros del río. El desplazamiento altitudinal evidenciado en este estudio se sustenta también con estudios que sugieren que los peces no nativos pueden modificar el comportamiento de las especies nativas, principalmente cuando llegan a ser numerosos, dominantes y/o más agresivos que las especies nativas (Cucherousset \& Olden, 2011).

La modificación en el comportamiento y/o la biología de las especies nativas se ha observado en cuerpos de agua invadidos por especies del género Poecilia, donde los machos intentan persistentemente forzar copulas con hembras de esas especies (Valero et al., 2008). Estos intentos de copulas forzadas acarrean costos para las hembras ya que pierden oportunidades de alimentarse, se exponen a la depredación e invierten energía en rechazarlos (González-Zuarth \& Vallarino, 2014), lo que a su vez podría representar costos muy altos e incluso riesgo de viabilidad para la población local (Valero et al., 2008) posiblemente provocando, como en este caso, un desplazamiento altitudinal.

Aunque son diversos los factores ambientales, físico-químicos y ecológicos, los que afectan la adopción y asignación de recursos energéticos para el crecimiento, la maduración y la reproducción (Enberg et al., 2012); es evidente que los parámetros ambientales registrados en el área de estudio no permiten identificar ninguna causalidad para la segregación altitudinal, ya que los rangos de tolerancia para los poecílidos como P. gillii (Chapman \& Chapman, 1993), sobrepasan los valores que aquí se han registrado; similar situación se presenta con $P$. fria que originalmente ocupaba todo el cauce, y lo sigue haciendo en todo el río Súa. Sin embargo, si los peces introducidos han ocupado con éxito la zona baja y media, están utilizando recursos que de otro modo serían utilizados por $P$. fria, esto podría verse expresado en cambios del tamaño total de la población reproductora nativa (Gebrekiros, 2016).

El crecimiento de los peces está directamente relacionado con la masa de la población, que es controlada por la reproducción e influenciado por el suministro de alimento (Nikolski, 1969). La mayor cantidad de proteína alimentaria se convierte en crecimiento y el consecuente aumento de la longitud antes de la madurez, posteriormente el alimento se utiliza principalmente para el aumento del peso y de las reservas para la migración reproductiva y el desarrollo de las gónadas (Gomiero, Carmassi, \& Braga, 2007). En muchas especies de peces las tasas de crecimiento varían proporcionalmente con las condiciones ambientales, la disponibilidad de alimento, y factores que afectan su utilización como la temperatura y la densidad poblacional (Gomiero et al., 2007), éstas tasas también pueden variar durante el ciclo de vida, estacionalmente y en cada hábitat ocupado por la especie (Lowe-McConnell, 1999). Aquí se evidencia una modificación en el crecimiento y la madurez de la población de P. fria del río Atacames (Tabla 3), que presenta una mayor tasa de crecimiento inicial, pero también una madurez más temprana, lo que detiene el crecimiento; al contrario de los que sucede con $P$. fria del río Súa, que presenta una menor tasa de crecimiento primario, pero demora un poco más su madurez, lo que también le permite alcanzar un mayor crecimiento; además, la edad de madurez calculada de $P$. 
gillii es similar a la de $P$. fria del río Atacames, aproximadamente en la edad " 8 ", pero en ambos casos es notoriamente anterior a la edad de madurez de $P$. fria del río Súa (Fig. 4). Esto puede ser debido a que cuando las especies no autóctonas se introducen en un nuevo entorno, las especies nativas enfrentan nuevas presiones de selección en las que deben demostrar una rápida adaptación para asegurar la supervivencia (Strauss, Lau, \& Carroll, 2006). Aunque las dietas descritas para $P$. gilli y $P$. fria en el río Atacames son mayoritariamente detritus, la primera especie alterna principalmente con algas filamentosas (más comunes en la zona media) mientras que la segunda con macroinvertebrados (más abundantes en la zona alta) (Vásquez, 2019), lo cual es comprensible dada la exclusión de esta última especie de la zona media en el río Atacames. Estos cambios adaptativos rápidos pueden llevar también a una modificación en los rasgos morfológicos de las especies nativas que reducen el riesgo de depredación (o la competencia) y la superposición de nichos con las especies invasoras, minimizando así el potencial de exclusión definitiva (Cucherousset \& Olden, 2011).

Mediante la morfometría geométrica se descarta la hibridación entre las dos especies, ya que su morfología muestra patrones claramente diferenciables; seguramente gracias a la trasmisión y recepción de señales intraespecíficas reguladas por las condiciones ecológicas locales (Morrongiello, Bond, Crook, \& Wong, 2010), lo que ha fortalecido la barrera reproductiva y ha impedido el apareamiento entre estas especies (González-Zuarth \& MacíasGarcía, 2006). Sin embargo, la morfometría evidencia también un cambio relevante en la forma del cuerpo de $P$. fria del Atacames, con respecto a $P$. fria del Súa el desplazamiento posterior de la aleta pectoral es particularmente notorio y significativo $(\mathrm{T}=219.44, \mathrm{P}<0.001)$ (Fig. 6); en términos generales, las aletas pares se ocupan de la producción de fuerzas verticales y por lo tanto afectan principalmente el equilibrio, particularmente en el ascenso y descenso (Harris, 1938). Se ha demostrado que esta aleta puede servir de freno, produciendo un levantamiento así como una fuerza de arrastre, la neutralización de esta fuerza de elevación por una fuerza hacia abajo producida por las aletas pélvicas requiere la migración hacia delante de esta última, si esta migración no tuvo lugar, los peces se inclinarían hacia arriba o subirían durante la parada (Lauder \& Drucker, 2004); es decir, que la migración de la aleta pectoral en $P$. fria del Atacames, (considerando que la posición de las aletas pélvicas se mantiene en la misma posición en ambas poblaciones), podría tener relación con una adaptación particular que favorezca la elevación, es decir los saltos. Soares y Bierman (2013) ya han registrado, para el caso de $P$. reticulata, la capacidad de saltar fuera del agua, que se repite localmente también en el caso de P. fria; Deacon y Magurran (2016) afirman que este tipo de saltos, más que una estrategia para evitar depredadores o capturar presas, posiblemente es un rasgo desarrollado para ayudar a la dispersión, es decir, un instinto que le facilita colonizar o encontrar nuevos cuerpos de agua; en este caso, tal vez facilitando el acceso a zonas altas del río a quienes poseían este fenotipo. Por otro lado, se ha documentado también el uso extensivo de las aletas pectorales como dispositivos primarios de locomoción y velocidad (Lauder \& Madden, 2006), por lo que además el posicionamiento posterior de esta aleta podría estar relacionado con una mejora en la movilidad y en la velocidad ante la presión ejercida por la otra especie.

Los elementos de cambio en la distribución del gradiente, en el crecimiento y en la forma del cuerpo evidenciados, podrían estar relacionados con una respuesta adaptativa producto de la plasticidad fenotípica ante la presencia de agentes externos (Fisk et al., 2007). Un número de estudios cada vez mayor demuestran que la capacidad de las poblaciones para experimentar una evolución contemporánea o rápida es posible (Swanne, Hendry, \& Reznick, 2017), este mismo autor menciona que esa plasticidad fenotípica puede influir en la dinámica evolutiva y, por lo tanto, en las tasas de evolución. El cambio en las características estaría vinculado en cierta medida con la historia evolutiva de las 
especies, pero se ha demostrado también que caracteres como el tamaño corporal y la forma del cuerpo son evolutivamente más probables de un cambio temprano (Blomberg, Garland, \& Ives, 2003).

La presencia de especies introducidas e invasoras en este tipo de ríos podría además generar cambios ecosistémicos que a futuro se expresarían en forma de cambio en los flujos biogeoquímicos, en la estructura trófica, en la estructura del hábitat (García-Berthou et al., 2015) $y / o$ en fuertes modificaciones de los ciclos de nutrientes (por ejemplo, nitrógeno y fósforo) desplazando la proporción relativa de cada nivel trófico (Eby, Roach, Crowder, \& Stanford, 2006). La identificación temprana de los cambios que la presencia de especies introducidas está generando en las especies locales es de gran importancia, para evitar que algún momento la pérdida de biodiversidad y la homogenización biótica (Olden, Lockwood, \& Parr, 2011) sea común en estos ambientes tropicales únicos.

Declaración de ética: los autores declaran que todos están de acuerdo con esta publicación y que han hecho aportes que justifican su autoría; que no hay conflicto de interés de ningún tipo; y que han cumplido con todos los requisitos y procedimientos éticos y legales pertinentes. Todas las fuentes de financiamiento se detallan plena y claramente en la sección de agradecimientos. El respectivo documento legal firmado se encuentra en los archivos de la revista.

\section{AGRADECIMIENTOS}

Este trabajo es un resultado de los proyectos con fondos internos de investigación de la Pontificia Universidad Católica del Ecuador Sede en Esmeraldas de los años 2017 y 2018; Los resultados de este trabajo forman parte de la tesis del primer autor dentro del Doctorado en Ciencias Agrarias y del Medio Natural de la Universidad de Zaragoza, con el apoyo del Banco Santander; agradecemos la colaboración en los muestreos de los estudiantes Michelle Mora y Josselyn Mera.

\section{RESUMEN}

Una de las causas que aportan a la disminución de la biodiversidad es la introducción de especies exóticas que muchas veces terminan siendo invasoras, y por lo tanto terminan compitiendo con especies locales. Esta competencia no siempre es equitativa ya que las especies invasoras comúnmente cuentan con ventajas adaptativas que le otorgan beneficios ante las especies locales. Este estudio realizado en microcuencas del noroccidente del Ecuador identifica los efectos de la reciente presencia de Poecilia gillii sobre la especie nativa Pseudopoecilia fria; mediante un análisis poblacional (distribución longitudinal, parámetros de crecimiento y morfometría). Los resultados muestran un desplazamiento de $P$. fria hacia la parte alta del río, un aceleramiento en su crecimiento y por lo tanto una madurez anticipada con la consecuente disminución del tamaño corporal y finalmente un cambio en el alto del cuerpo con deslizamiento posterior de la aleta pectoral.

Palabras clave: especie invasora, Pacífico, impacto.

\section{REFERENCIAS}

Balzarini, M., Gonzalez, L., Tablada, M., Casanoves, F., Di Rienzo, J., \& Robledo, C. W. (2008). Infostat. Manual del Usuario. Córdoba, Argentina: Editorial Brujas.

Barquero-González, J., Mora-Vargas, J., Valerio-Vargas, J., \& Ulloa-Rojas, J. (2018). Diversidad de la ictiofauna de la laguna Hule, Costa Rica. UNED Research Journal, 10(1), 214-220.

Blomberg, S., Garland, T., \& Ives, A. (2003). Testing for phylogenetic signal in comparative data: behavioral traits are more labile. Evolution, 57, 717-745.

Bourke, P., Magnan, P., \& Rodriguez, M. (1999). Phenotypic responses of lacustrine brook charr in relation to the intensity of interspecific competition. Evolutionary Ecology, 13, 19-31.

Bussing, W. (1998). Peces de las aguas continentales de Costa Rica (Freshwater fishes of Costa Rica) ( $2^{\mathrm{a}}$ ed.). San José Costa Rica: Editorial de la Universidad de Costa Rica.

Camacho-Cervantes, M., García, C., Ojanguren, A. F., \& Magurran, A. E. (2014a). Exotic invaders gain foraging benefits by shoaling with native fish. Royal Society Open Science, 1(3). DOI: 10.1098/ rsos.140101

Camacho-Cervantes, M., Ojanguren, A., Deacon, A., Ramnarine, I., \& Magurran, A. (2014b). Association 
tendency and preference for heterospecifics in an invasive species. Behaviour, 151, 769-780.

Camacho-Cervantes, M., Ojanguren, A., DomiínguezDomínguez, O., \& Magurran, A. (2018). Sociability between invasive guppies and native topminnows. PLoS ONE, 13(2), e0192539.

Camacho-Cervantes, M., Ojanguren, A., \& Magurran, A. (2015). Exploratory behaviour and transmission of information between the invasive guppy and native Mexican topminnows. Animal Behaviour, 106, 115-20.

Chapman, L., \& Chapman, C. (1993). Desiccation, flooding, and the behavior of Poecilia gillii (Pisces: Poeciliidae). Ichthyological Explorer Freshwaters, 4(3), 279-287.

Courtenay, W. R., \& Meffe, G. K. (1989). Small fishes in strange places: a review of introduced poeciliids. In G. K. Meffe \& F. F. Snelson (Eds.), Ecology and Evolution of Livebearing Fishes (Poeciliidae) (pp. 319-331). New Jersey: Prentice Hall.

Cucherousset, J., \& Olden, J. D. (2011). Ecological impacts of non-native freshwater fishes. Fisheries, $36,215-230$.

Deacon, A. E., \& Magurran, A. E. (2016). How Behaviour Contributes to the Success of an Invasive Poeciliid Fish: The Trinidadian Guppy (Poecilia reticulata) as a model Species. In J. S. Weis \& D. Sol (Eds.), Biological Invasions and Animal Behaviour (pp. 266-290). USA: Cambridge University Press.

Deacon, A. E., Ramnarine, I. W., \& Magurran, A. E. (2011). How Reproductive Ecology Contributes to the Spread of a Globally Invasive Fish. PLoS ONE, $6(9)$, e24416.

Di Rienzo, J. A., Casanoves, F., Balzarini, M. G., Gonzalez, L., Tablada, M., \& Robledo, C. W. (2019). InfoStat (versión 2019). Centro de Transferencia InfoStat, FCA, Universidad Nacional de Córdoba, Argentina. Recuperado de http://www.infostat.com.ar

Eby, L. A., Roach, W. J., Crowder, L. B., \& Stanford, J. A. (2006). Effects of stocking-up freshwater food webs. Trends in Ecology and Evolution, 21, 576-584.

Enberg, K., Jørgensen, C., Dunlop, E. S., Varpe, Ø., Boukal, D. S., Baulier, L., ... Heino, M. (2012). Fishinginduced evolution of growth: concepts, mechanisms and the empirical evidence. Marine Ecology, 33, $1-25$.

Fisk, D. F., Latta, L. C., IV, Knapp, R. A., \& Pfrender, M. E. (2007). Rapid evolution in response to introduced predators I: rates and patterns of morphological and life-history trait divergence. BMC Evolutionary Bio$\log y, 7,22$
Froese, R., \& Pauly, D. (2018). FishBase. World Wide Web electronic publication [Poecilia gillii]. Retrieved from http://www.fishbase.org/summary/Poeciliagillii.html

García-Berthou, E., Almeida, D., Benejam, L., Magellan, K., Bae, M. J., Casals, F., \& Merciai, R. (2015). Impacto ecológico de los peces continentales introducidos en la península ibérica. Ecosistemas, 24, 36-42.

Gayanilo, F. C. Jr., Sparre, P., \& Pauly, D. (1996). The FAOICLARM Stock Assessment Tools (FiSAT) User's Guide. FAO Computerized Information Series, No. 8 (Fisheries). FAO, Roma.

Gebrekiros, S. T. (2016). Factors Affecting Stream Fish Community Composition and Habitat Suitability. Journal of Aquaculture \& Marine Biology, 4(2), 00076.

Gomiero, L. M., Carmassi, A. L., \& Braga, F. M. S. (2007). Crescimento e mortalidade de Brycon opalinus (Characiformes, Characidae) no Parque Estadual da Serra do Mar, Mata Atlântica, Estado de São Paulo. Biota Neotropica, 7(1), 22-26.

González-Zuarth, C., \& Macías-García, C. (2006). Phenotypic differentiation and pre-mating isolation between allopatric populations of Girardinichthys multiradiatus. Proceedings of the Royal Society of London Series B, 273, 301-307.

González-Zuarth, C. \& Vallarino, A. (2014). El impacto de los peces invasores sobre la comunicación entre los sexos ¿una posible vía hacia la extinción? Una revisión. En A. Low-Pfeng, P. Quijón, \& E. PetersRecagno (Eds.), Especies invasoras acuáticas: casos de estudio en ecosistemas de México (pp. 143-176). Ciudad de México, México: Secretaría de Medio Ambiente y Recursos Naturales (Semarnat), Instituto Nacional de Ecología y Cambio Climático (INECCSemarnat) \& University of Prince Edward Island (UPEI).

Gubiani, E., Ruaro, E., Ribeiro, V., Algeri-Eichelberger, A., Bogoni, R., Dorigon-Lira, A., ... da Graça, W. (2018). Non-native fish species in Neotropical freshwaters: how did they arrive, and where did they come from? Hydrobiologia, DOI: 10.1007/ s10750-018-3617-9

Harris, J. E. (1938). The role of the fi ns in the equilibrium of the swimming fi sh. II. The role of the pelvic fins. Journal of Experimental Biology, 15, 32-47.

Jiménez-Prado, P. (2012). Contaminación del río Atacames y su impacto en la comunidad de peces. Anuario de Investigación y Desarrollo. Ecuador: Centro de Investigación y Desarrollo de la Pontificia Universidad Católica del Ecuador Sede Esmeraldas.

Klingenberg, C. P. (2011). MorphoJ: an integrated software package for geometric morphometrics. Molecular Ecology Resources, 11, 353-357. 
Lampert, K. P., \& Schartl, M. (2008). The origin and evolution of a unisexual hybrid: Poecilia formosa. Philosophical Transactions of the Royal Society B., 363(1505), 2901-09.

Latta, L. C., IV, Bakelar, J. W., Knapp, R. A., \& Pfrender, M. E. (2007). Rapid evolution in response to introduced predators II: the contribution of adaptive plasticity. BMC Evolutionary, 7, 21.

Lauder, G. V., \& Drucker, E. G. (2004). Morphology and Experimental Hydrodynamics of Fish Fin Control Surfaces. IEEE Journal of Oceanic Engineering, 29(3), 556-571.

Lauder, G. V., \& Madden, P. (2006). Learning from fish: kinematics and experimental hydrodynamics for roboticists. International Journal of Automation and Computing, 4, 325-335.

Leimar, O., \& Hammerstein, P. (2010). Cooperation for direct fitness benefits. Royal Society Philosophical Transactions Biological Sciences, 365(1553), 2619-2626.

López-Sepulcre, A., Gordon, S. P., Paterson, I. G., Bentzen, P., \& Reznick, D. N. (2013). Beyond lifetime reproductive success: the posthumous reproductive dynamics of male Trinidadian guppies. Proceedings of the Royal Society B: Biological Sciences, 280(1763), 20131116.

Lowe-McConnell, R. H. (1999). Estudos ecológicos de comunidades de peixes tropicais. São Paulo: da Universidade de São Paulo.

Magalhães, A. L. B., \& Jacobi, C. M. (2017). Colorful invasion in permissive Neotropical ecosystems: establishment of ornamental non-native poeciliids of the genera Poecilia / Xiphophorus (Cyprinodontiformes: Poeciliidae) and management alternatives. Neotropical Ichthyology, 15, e160094.

Magurran, A. E. (2009). Threats to Freshwater Fish. Science, 325(5945), 1215-1216.

Magurran, A. E., Seghers, B. H., Carvalho, G. R., \& Shaw, P. W. (1992). Behavioral Consequences of an Artificial Introduction of Guppies (Poecilia reticulata) in N-Trinidad - Evidence for the Evolution of Antipredator Behavior in the Wild. Proceedings of the Royal Society of London Series B-Biological Sciences, 248, 117-122.

Mathis, A., \& Chivers, D. P. (2003). Overriding the oddity effect in mixed-species aggregations: group choice by armored and nonarmored prey. Behavioral Ecology, 14(3), 334-339.

Mena, F., Fernández San Juan, M., Campos, B., SánchezÁvila, J., Faria, M., \& Pinnock, M. (2014) Pesticide residue analyses and biomarker responses of native Costa Rican fish of the Poeciliidae and Cichlidae families to assess environmental impacts of pesticides in Palo Verde National Park. Journal of Environmental Biology, 35(1), 19-27.

Meyer, A., Salzburger, W., \& Schartl, M. (2006). Hybrid origin of a swordtail species (Teleostei: Xiphophorus clemenciae) driven by sexual selection. Molecular Ecology, 15(3), 721-30.

Montaño, H. (2018). Comparación de características geomorfológicas de las cuencas del río Atacames y Súa (Tesis de grado). PUCESE, Esmeraldas.

Morales, J. \& García-Alzate, C. (2016). Estructura trófica de los peces en arroyos del Corral de San Luis, cuenca del Bajo Magdalena, Caribe, Colombia. Revista de Biología Tropical, 64(2), 715-732.

Morrongiello, J. R., Bond, N. R., Crook, D. A., \& Wong, B. B. M. (2010). Nuptial coloration varies with ambient light environment in a freshwater fish. Journal of Evolutionary Biology, 23, 2718-2725.

Nikolskii, G. V. (1969). Theory of Fish Population Dynamics as the Biological Background for Rational Exploitation and Management of Fishery Resources. Edimburgo, Escocia: R. Jones.

Olden, J. D., Lockwood, J. L., \& Parr, C. L. (2011). Biological invasions and the homogenization of faunas and floras. En R. J. Ladle \& R. J. Whittaker (Eds.), Conservation Biogeography (pp. 224243). Oxford, Inglaterra: Blackwell Publishing Ltd.

Ortega, H., Guerra, H., \& Ramírez, R. (2007). The introduction of nonnative fishes into freshwater systems of Peru. In T. M. Bert (Ed.), Ecological and genetic implications of aquaculture activities (pp 247-278). Dordrecht, Netherlands: Springer.

Pauly, D. (1979). Theory and management of tropical multispecies stocks-A review, with emphasis on the Southeast Asia demersal fisheries. ICLARM Studies and Reviews, 1, 1-35.

Pauly, D. (1983). Some simple methods for the assessment of tropical fish stocks. FAO Fisheries Technical Paper, 234, 1-52.

Pauly, D., \& David, N. (1981). ELEFAN I, a BASIC program for the objective extraction of growth parameters from length-frequency data. Meeresforschung, 28(4), 205-211.

Pauly, D., \& Munro, J. L. (1984). Once More on the Comparison of Growth in Fish and Invertebrates. ICLARM Fishbyte, 2, 21.

Rodríguez-Pérez, B. \& Contreras, E. (2012). Evaluación de la diversidad ictiofaunística del río Estelí Nicaragua. Revista Científica FAREM-Esteli, 1, 1-24.

Rohlf, F. J. (2018a). TpsUtil (Version 1.76). Retrieved from http://life.bio.sunysb.edu/morph 
Rohlf, F. J. (2018b). TpsDig2 (Version 2.31). Retrieved from http://life.bio.sunysb.edu/morph

Rohlf, F. J. (2018c). TpsRelw32 (Version 1.53). Retrieved from http://life.bio.sunysb.edu/morph

Shea, K., \& Chesson, P. (2002). Community ecology theory as a framework for biological invasions. Trends in Ecology \& Evolution, 17(4), 70-76.

Soares, D., \& Bierman, H. S. (2013). Aerial jumping in the Trinidadian guppy (Poecilia reticulata). PLoS ONE, $8, \mathrm{e} 61617$.

Sparre, P., \& Venema, S. C. (1992). Introduction to tropical fish stock assessment. Part 1. FAO Fisheries Technical Paper, 306, 1-376.

Stephens, P. A., \& Sutherland, W. J. (1999). Consequences of the Allee effect for behaviour, ecology and conservation. Trends in Ecology \& Evolution, 14(10), 401-405.

Strauss, S. Y., Lau, J. A., \& Carroll, S. P. (2006). Evolutionary responses of natives to introduced species: what do introductions tell us about natural communities? Ecology Letters, 9, 357-374.

Swanne, P. G., Hendry, A. P., \& Reznick D. N. (2017). Predator-induced Contemporary Evolution, Phenotypic
Plasticity, and the Evolution of Reaction Norms in Guppies. Copeia, 105(3), 514-522.

Taylor, C. M., \& Hastings, A. (2005). Allee effects in biological invasions. Ecology Letters, 8(8), 895-908.

Thompson, D. W. (1917). On growth and form. London: Cambridge University Press.

Toro, M. V., Manríquez, G., \& Sauzo, I. (2010). Morfometría geométrica y el estudio de las formas biológicas: De la morfología descriptiva a la morfología cuantitativa. International Journal of Morphology, 28(4), 977-990.

University of Michigan Museum of Zoology (2018). University of Michigan museum of zoology, division of fishes (Version 1.3). occurrence dataset DOI: $10.15468 / 8 \mathrm{cxijb}$

Valero, A., Macías-García, C., \& Magurran, A. E. (2008). Heterospecific harassment of native endangered fishes by invasive guppies in Mexico. Biology Letters, 4(2), 149-152.

Vásquez, W. F. (2019). Contenido estomacal y diferenciación sexual de peces de los ríos Súa y Atacames (Tesis de grado). PUCESE, Esmeraldas.

Williamson, M. H. \& Fitter, A. (1996). The varying success of invaders. Ecology, 77, 1661-1666. 